\title{
SUCCESSIVE ADDITION OF DIGITS OF AN INTEGER NUMBER: ON PROPERTIES AND ROLE IN STUDYING DISTRIBUTION OF PRIMES
}

\author{
Manish Khare*, Assistant Professor in Physics, MIT Academy of Engineering, \\ Alandi(D), PUNE -412105 INDIA Email address:- $\underline{\text { mrkhare@ esci.maepune.ac.in }}$
}

Kalyanlakshmi Chitta, Manager, Union Bank of India, Alandi(D), PUNE-412105 INDIA Email address:- kalyanilakshmichitta@gmail.com

\begin{abstract}
:
Successive-addition-of-digits-of-a-number(SADN) refers to the process of adding up the digits of an integer number until a single digit is obtained. Concept of SADN has been occasionally identified but seldom employed in extensive mathematical applications. This paper discusses SADN and its properties in terms of addition, subtraction and multiplication. Further, the paper applies the multiplication-property of SADN to understand the distribution of prime numbers. For this purpose the paper introduces three series of numbers -S1, S3 and S5 series- into which all odd numbers can be placed, depending on their SADN and the rationale of such classification. Extending the analysis the paper explains how composite numbers of the S1 and S5 series can be derived. Based on this discussion it concludes that even as the concept of SADN is rather simple in its formulation and appears as an obvious truism but a profound analysis of the properties of SADN in terms of fundamental mathematical functions reveals that SADN holds a noteworthy position in number theory and may have significant implications for unfolding complex mathematical questions like understanding the distribution of prime numbers and Goldbachproblem.
\end{abstract}

Keywords: Primes, Distribution of Primes, Primes and integers, Additive questions involving primes, Number representations 


\section{Section-I: Introduction}

Present paper discusses a mathematical function which has been occasionally identified but seldom employed to extensive application. This function is termed as Successive Addition of Digits of (integer) Number and is acronymed as SADN function. This paper studies the fundamental properties of SADN function and its behaviour under algebraic operations of addition, multiplication and subtraction. The paper further discusses the application of the multiplication property of SADN in understanding the distribution of prime numbers. Apart from this, present paper also discusses the distribution of primes. An extension of present paper is to explore the possibilities of application of select properties of SADN function in an attempt to discuss well-established and centuries-old conjectures.

This article is organized in the following manner- The present section (Section 1) is introductory in nature and sets out the basic areas of inquiry that the paper seeks to address. Section 2 introduces the concept of Successive addition of digits of a number and discusses the fundamental properties of SADN in terms of basic mathematical functions like addition, subtraction and multiplication. Section 3 discusses the SADN of prime numbers. Section 4 introduces three series of numbers - the S1, S3 and S5 series-into which all odd numbers can be placed, depending on their SADN alongwith the rationale for making such a classification. Section 5 applies the multiplication property of SADN to discuss why the S3 series introduced in Section 4 comprises exclusively of composite numbers while the S1 and S5 series comprise of both prime and composite numbers. Section 6 discusses how composite numbers on the S1 and S5 series can be derived. Section 7 is in the nature of a brief conclusion.

\section{Section-II}

- What is SADN?

- What are the properties of SADN?

- Proof of the properties of SADN

The function of SADN stands for Successive Addition of Digits of (integer) Number. SADN function exhibits following properties:-

\section{Idempotence}




\author{
II. Range of SADN function \\ III. Distribution over addition \\ IV. Distribution over multiplication \\ V. Additive Identity for SADN function \\ VI. Interchangeability of non-positive SADN and positive SADN \\ VII. Distribution over subtraction \\ VIII. Multiplicative Identity for SADN function
}

\title{
I. Property of Idempotence:-
}

SADN function stands for successive-addition-of-digits-of-number. Addition is an operation which is operated upon multiple operands and not defined in case of a single operand alone. Here the term 'successive' implies that the digits of the number are to be added as long as the operation of addition is defined. It implies that to determine SADN of any given number, its digits are to be successively added until a single digit is obtained. This single digit is termed as SADN of the given number.

Example: Suppose the given number is 546289 . Addition of its digits $=5+4+6+2+8+9=34$

Successive addition of digits $=3+4=7$

In our example, $\operatorname{SADN}(546289)=7$

In general terms: $\operatorname{SADN}(\mathrm{x})=\operatorname{SADN}(\operatorname{SADN}(\operatorname{SADN}(\ldots \operatorname{SADN}(\mathrm{x}))))$ which says that $\operatorname{SADN}$ function is an idempotent function.

\section{Range of SADN function:-}

The property of idempotence implies that the value of SADN function for any non-zero integer number would be a single digit integer only ranging from 1 to 9.

$1 \leq \mathrm{SADN}(\mathrm{x}) \leq 9$ implies that $\operatorname{SADN}(\mathrm{x})=\{1,2,3,4,5,6,7,8,9\}$

\section{III.Property of distribution over addition:-}


$\operatorname{SADN}(x+y)=\operatorname{SADN}(x)+\operatorname{SADN}(y)$

SADN function is distributive over addition.

The paper identifies the phenomenon of SADN as analogous to 'valency of an atom'. In case of an atom, its inner shell gets filled by as much number of electrons as suggested by the octet rule and the outermost shell is known as valence shell. Electrons occupying the valence-shell are called as valence-electrons. Just as valence-electrons are responsible for the properties exhibited by the corresponding atom, the current row plays a central role in portraying the properties of SADN of an integer.

Consider the natural numbers written in matrix form where number of rows is nine (9) and number of columns goes on increasing. Upon writing in this form, we get a sample matrix M1 for natural numbers from one(1) to fifty(50) as following:-

$\begin{array}{lllllll}\text { Row(1) having elements of SADN(1): } & 1 & 10 & 19 & 28 & 37 & 46 \\ \text { Row(2) having elements of SADN(2): } & 2 & 11 & 20 & 29 & 38 & 47 \\ \text { Row(3) having elements of SADN(3): } & 3 & 12 & 21 & 30 & 39 & 48 \\ \text { Row(4) having elements of SADN(4): } & 4 & 13 & 22 & 31 & 40 & 49 \\ \text { Row(5) having elements of SADN(5): } & 5 & 14 & 23 & 32 & 41 & 50 \\ \text { Row(6) having elements of SADN(6): } & 6 & 15 & 24 & 33 & 42 & \\ \text { Row(7) having elements of SADN(7): } & 7 & 16 & 25 & 34 & 43 & \\ \text { Row(8) having elements of } \operatorname{SADN}(8): & 8 & 17 & 26 & 35 & 44 & \\ \text { Row(9) having elements of } \operatorname{SADN}(9): & 9 & 18 & 27 & 36 & 45 & \end{array}$

\section{Matrix M1}

Through this matrix M1, we discuss about SADN as follows:

In order to determine the SADN of an integer ' $n$ '; we write numbers, starting from one(1), till the integer ' $n$ '. The column in which the integer ' $n$ ' exists, is called as current column. All columns filled before the current column are known as complete columns. For determining SADN of ' $n$ '; we don't bother about the number of complete columns and count the number of rows of the current column. Number of row in which integer(n) exists; is known as SADN of the integer(n). In this way, the current column in case of SADN is similar to what valence-shell is in case of an atom.

\section{Property of distribution over addition:}


SADN of $(x+y)=\operatorname{SADN}$ of $(x)+\operatorname{SADN}$ of $(y)$

SADN function is distributive over addition.

\section{Proof:-}

SADN of integers $\mathrm{x}$ and $\mathrm{y}$ denote the number of rows of current columns corresponding to $\mathrm{x}$ and $\mathrm{y}$ as mentioned in matrix M1. In case of addition: Suppose SADN of $\mathrm{x}$ is given as $\mathrm{x}$ ' and SADN of $y$ is given as $y^{\prime}$. Here $x^{\prime}$ and $y^{\prime}$ would be natural numbers such that the value of $x^{\prime}$ and $y^{\prime}$ cannot exceed the number 9; as there are only 9 rows in matrix M1. Either x' $+y$ ' would be $<=9$ (i.e. first case) or it would be $>9$ (i.e. second case) but can never be greater than 18 , which is the case if both x' and y' attain their maximum possible values; i.e. 9.

\section{In first case when $x^{\prime}+y^{\prime}$ would be $<=9$,}

If we write numbers upto $\mathrm{x}$ as per matrix M1 and call this arrangement as matrix M1x, we may get some number of complete columns alongwith x' rows in current column and upon writing numbers upto $\mathrm{y}$ and call it matrix M1y, we may get some number of complete columns alongwith y' rows in current column. Now if we write numbers upto $\mathrm{x}+\mathrm{y}$ as matrices $\mathrm{M} 1 \mathrm{x}$ and M1y standing side by side, the number of complete columns remains as summation of number of complete columns of M1x and M1y. In present case the summation of number of filled rows (i.e. $\left.x^{\prime}\right)$ in current column of M1 $x$ and that of in M1y gives us $x^{\prime}+y^{\prime}$. As $x^{\prime}+y^{\prime}<=9$, it implies that no extra complete column is generated upto the integer $\mathrm{x}+\mathrm{y}$.

Hence $\operatorname{SADN}$ of $(\mathrm{x}+\mathrm{y})=\mathrm{SADN}$ of $(\mathrm{x})+\mathrm{SADN}$ of $(\mathrm{y})$ in first case.

\section{In second case when $x^{\prime}+y^{\prime}$ would be $>9$,}

If we write numbers upto $\mathrm{x}$ as per matrix M1 and call this arrangement as matrix M1x, we may get some number of complete columns alongwith x' rows in current column and upon writing numbers upto $\mathrm{y}$ and call it matrix M1y, we may get some number of complete columns alongwith y' rows in current column. Now if we write numbers upto $\mathrm{x}+\mathrm{y}$ as matrices M1x and M1y standing side by side, the number of complete columns remains, (till now) as addition of number of complete columns of M1x and M1y. In present case the summation of number of filled rows (i.e. $x^{\prime}$ ) in current column of M1x and that of in M1y gives us $x^{\prime}+y^{\prime}$. As $x^{\prime}+y^{\prime}>9$, it 
implies that one extra complete column is generated if we write numbers upto the integer $\mathrm{x}+\mathrm{y}$ as arranged in matrix M1. As number of rows in this newly generated complete column would be 9 only; number of filled rows of current column would become x'+y'-9. As in present case, $9<$ $\mathrm{x}^{\prime}+\mathrm{y}^{\prime}<=18$; there may be total of 9 subcases, corresponding to $\mathrm{x}^{\prime}+\mathrm{y}^{\prime}$ as any one of 10 or 11 or 12 or 13 or 14 or 15 or 16 or 17 or 18 . In these subcases the corresponding row number of current column would be 1 or 2 or 3 or 4 or 5 or 6 or 7 or 8 or 9 . As 1 to 9 are SADN of 10 to SADN of 18 respectively i.e. 10 is the $1^{\text {st }}$ number after removing initial 9 numbers and similarly 18 is the $9^{\text {th }}$ number after removing initial 9 numbers. Hence in this case as well, if numbers upto $x+y$ are arranged in terms of matrix M1 and this arrangement is denoted as M1(x+y), then SADN of $(x+y)$ is summation of individual SADNs of the integers $x$ and $y$.

Hence SADN of $(x+y)=$ SADN of $(x)+$ SADN of $(y)$ in second case as well.

Abovementioned analysis directs us to say that in any of the possible cases:

SADN of $(x+y)=\operatorname{SADN}$ of $(x)+\operatorname{SADN}$ of $(y)$

i.e. SADN function is distributive over addition.

Example: $\operatorname{SADN}(28)+\operatorname{SADN}(541)=\operatorname{SADN}(28+541)=\operatorname{SADN}(569)$

Under property of idempotence $\operatorname{SADN}(569)=\operatorname{SADN}(20)=2$

\section{IV.Property of distribution over multiplication:-}

SADN of $(x \cdot y)=$ SADN of $(x) \cdot S A D N$ of $(y)$

SADN function is distributive over multiplication.

\section{Proof:}

Suppose SADN of $\mathrm{x}$ is given as $\mathrm{x}^{\prime}$ and SADN of $\mathrm{y}$ is given as $\mathrm{y}$.

In case $\mathrm{SADN}$ of $(\mathrm{x} . \mathrm{y})=\mathrm{SADN}$ of $(\mathrm{x}+\mathrm{x}+\mathrm{x}+\ldots \mathrm{y}$ times $)=\mathrm{SADN}$ of $\mathrm{x}+\mathrm{SADN}$ of $\mathrm{x}+\ldots \mathrm{y}$ times $=\mathrm{y} \cdot[\operatorname{SADN}$ of $(\mathrm{x})]=\mathrm{y} \cdot \mathrm{x}$ '

[by property of distribution over addition] 
Implies that SADN of $(x . y)=y \cdot x^{\prime}$

Property of idempotence of SADN function says that:-

$\operatorname{SADN}$ of $(\mathrm{x} \cdot \mathrm{y})=\mathrm{SADN}$ of $[\operatorname{SADN}$ of $(\mathrm{x} \cdot \mathrm{y})]=\mathrm{SADN}$ of $\left(\mathrm{y} \cdot \mathrm{x}^{\prime}\right)=\mathrm{x}^{\prime} \cdot \mathrm{y}^{\prime}$ i.e. [SADN of $\left.(\mathrm{x})\right]$. [SADN of $(y)]$

Implies that SADN of $(x . y)=[\operatorname{SADN}$ of $(x)] .[\operatorname{SADN}$ of $(y)]$

Example: SADN(12) . SADN(15)= SADN(12.15)=SADN(180)

Under property of idempotence: $\operatorname{SADN}(180)=9$

\section{Additive Identity for SADN function:-}

As SADN is primarily a type of addition operator, additive identity zero(0) acts as an additive identity for SADN as well.

Apart from zero, the number nine(9) also acts as an additive identity for SADN function.

\section{Proof:}

The reason of 9 being an additive identity for SADN function is as follows:-

In context of arranging the integers as per the matrix M1, SADN of any integer is identified by the number of row in which that integer lies in current column. Placing the digit 9 anywhere in an integer changes the integer in such a way that the new integer has more number of complete columns of 9 numbers and the number of filled rows in current column remains unchanged. As the number of filled rows of current column denotes the SADN of integer, hence introduction of digit 9 within the integer doesn't affect the SADN of that integer. This drives us to conclude that the number 9 acts as additive identity for SADN function.

Example: $\operatorname{SADN}(52)=\operatorname{SADN}(529)=\operatorname{SADN}(5092990)=7$ 


\section{Interchangeability of non-positive SADN and positive SADN:-}

Properties of distribution over addition and identity of SADN function leads to following equivalence between nonpositive and positive values of SADN

\begin{tabular}{|l|l|l|l|l|l|l|l|l|l|}
\hline $\begin{array}{l}\text { Positive } \\
\text { SADN digit }\end{array}$ & 1 & 2 & 3 & 4 & 5 & 6 & 7 & 8 & 9 \\
\hline $\begin{array}{l}\text { Equivalent } \\
\text { non-positive } \\
\text { SADN digit }\end{array}$ & -8 & -7 & -6 & -5 & -4 & -3 & -2 & -1 & 0 \\
\hline
\end{tabular}

\section{Table-1: Equivalence between nonpositive and positive values of SADN}

Both numbers of same columns are considered to be identical and replaceable substitutes of one another if need arises to consider negative digit for SADN.

This property of interchangeability relates negative and positive SADN as follows:

$$
\operatorname{SADN}(-\mathrm{a})=-\operatorname{SADN}(\mathrm{a})
$$

\section{Distribution over subtraction:-}

Under application of Table1:- SADN(x)-SADN(y)= SADN(x-y)

$\operatorname{SADN}(x)-\operatorname{SADN}(y)-\operatorname{SADN}(z)=\operatorname{SADN}(x-y-z)=\operatorname{SADN}(x)-\operatorname{SADN}(y+z)$

Example1: $\quad \operatorname{SADN}(724-452)=\operatorname{SADN}(724)-\operatorname{SADN}(452)=\operatorname{SADN}(4)-\operatorname{SADN}(2)=\operatorname{SADN}(2)$

$=2$

OR $\quad \operatorname{SADN}(724-452)=\operatorname{SADN}(724)-\operatorname{SADN}(452)=\operatorname{SADN}(4)-\operatorname{SADN}(2)=\operatorname{SADN}(4)$ $+\operatorname{SADN}(-2)=\operatorname{SADN}(4)+\operatorname{SADN}(7)=\operatorname{SADN}(11)=2($ refer table 1$)$

And $\operatorname{SADN}(724-452)=\operatorname{SADN}(272)=\operatorname{SADN}(2)=2$ 
Example2: $\quad \operatorname{SADN}(121-24)=\operatorname{SADN}(121)-\operatorname{SADN}(24)=\operatorname{SADN}(4)-\operatorname{SADN}(6)=-2=7($ refer table 1)

$$
\begin{aligned}
& \text { OR } \begin{array}{l}
\operatorname{SADN}(121-24) \quad=\quad \operatorname{SADN}(121)-\operatorname{SADN}(24)=\operatorname{SADN}(4)-\operatorname{SADN}(6) \\
=\operatorname{SADN}(4)+\operatorname{SADN}(-6)=\operatorname{SADN}(4)+\operatorname{SADN}(3)=\operatorname{SADN}(7)=7(\text { refer table } 1)
\end{array} \\
& \text { And } \operatorname{SADN}(121-24)=\operatorname{SADN}(97)=7
\end{aligned}
$$

\section{Multiplicative Identity for SADN function:-}

Apart from SADN(1) as multiplicative identity for every SADN function; following are multiplicative identities as special cases:-

$\operatorname{SADN}(4,7,1)$ acts as multiplicative identities for $\operatorname{SADN}(3,6)$

$\operatorname{SADN}(1,2,3,4,5,6,7,8,9)$ or $\operatorname{SADN}(n)$ act as multiplicative identity for $\operatorname{SADN}(9)$

\section{Section-III: Prime numbers and their SADN}

- SADN of Primes

Prime numbers are a particular type of subset of natural numbers. Any prime number would be divisible by itself and by the number 1 only. Primes cannot be divided by any other natural number except themselves and 1. In terms of SADN function, the divisibility test of 3 says that any natural number would be divisible by 3 only if its SADN is 3,6 or 9. It leads to conclude that any natural number whose $\mathrm{SADN}$ is 3,6 or 9 would be a composite as it would be divisible by the number 3 , hence SADN of primes can never be 3,6 or 9 (only exception to this would be number ' 3 ' itself). This discussion in conjunction with the properties of range of SADN says that $\mathrm{SADN}$ of primes may be $1,2,4,5,7$ or 8 .

If ' $\mathrm{P}$ ' represents a prime number, then $\operatorname{SADN}(\mathrm{P})=\{1,2,4,5,7,8\}$ 


\section{Section-IV Of composites and primes: The three series of odd numbers; not so 'odd'}

- The three (3) series of odd numbers-S1,S3 and S5 series

- Logic behind the three series

- Why S3 series will have only composite numbers (with only exception of the number3)

- S1 and S5 series will have both primes and composites

Set of natural numbers $N=\{1,2,3,4,5,6,7,8,9,10,11, \ldots \ldots .$.

$\mathrm{N}=\mathrm{E}+\mathrm{D}=\mathrm{E}+\{1\}+\mathrm{S} 1+\mathrm{S} 3+\mathrm{S} 5$

Where $\mathrm{E}$ is set of even numbers, $\mathrm{E}=\{2,4,6,8,10, \ldots\}$

And $\mathrm{D}=$ set of odd numbers $=\{1\}+\mathrm{S} 1+\mathrm{S} 3+\mathrm{S} 5=\{3,5,7,9,11, \ldots\}$

All elements of the set of even numbers ' $E$ ' are composites (with exception of the number 2) whereas elements of the set of odd numbers ' $\mathrm{D}$ ' may be prime or composite.

Now consider the following three series of odd numbers:-

$\mathrm{S} 1=\mathrm{a} 1+6 \mathrm{n}$ where $\mathrm{a} 1$ is 1 and $\mathrm{n}$ is a natural number implying

$\mathrm{S} 1=1+6 \mathrm{n} ; \mathrm{n} \in\{\mathrm{N}\}$

$\mathrm{S} 1=\{7,13,19,25,31,37,43,49,55,61,67, \ldots .$.

$\operatorname{SADN}($ element of $\mathrm{S} 1)=\{1,7,4\}$ in cyclic order

$\operatorname{SADN}(a 1+6 n) \in \mathrm{S} 1$ implies that $\operatorname{SADN}(1+6 n) \in \mathrm{S} 1$

$\mathrm{S} 3=\mathrm{a} 3+6 \mathrm{n}$ where $\mathrm{a} 3$ is 3 and $\mathrm{n}$ is a natural number implying

$\mathrm{S} 3=\{3,9,15,21,27,33,39,45,51,57,63,69, \ldots .$.

SADN(element of S3) $=\{3,9,6\}$ in cyclic order

$\operatorname{SADN}(a 3+6 n) \in S 3$ implies that $\operatorname{SADN}(3+6 n)$ e S3 
$\mathrm{S} 5=\mathrm{a} 5+6 \mathrm{n}$ where a5 is 5 and $\mathrm{n}$ is a natural number implying

$\mathrm{S} 5=6 \mathrm{n}-1 ; \mathrm{n} \in\{\mathrm{N}\}$

$\mathrm{S} 5=\{5,11,17,23,29,35,41,47,53,59,65,71, \ldots$.

$\operatorname{SADN}($ element of S5) $=\{5,2,8\}$ in cyclic order

$\mathrm{SADN}(\mathrm{a} 5+6 \mathrm{n}) \in \mathrm{S} 5$ implies that $\mathrm{SADN}(5+6 \mathrm{n}) \in \mathrm{S} 5$ implies that $\mathrm{SADN}(6 \mathrm{n}-1) \in \mathrm{S} 5$

\begin{tabular}{|l|l|}
\hline SADN $(\mathrm{n})=$ & $\mathrm{n} \epsilon$ \\
\hline $1,7,4$ & $\mathrm{~S} 1$ \\
\hline $3,9,6$ & $\mathrm{~S} 3$ \\
\hline $5,2,8$ & $\mathrm{~S} 5$ \\
\hline
\end{tabular}

Table2: SADN of a number determines its Series out of S1, S3, S5

Such segregation of odd numbers in term of three series S1, S3 and S5 leads to segregation of primes. As series $S 3$ consists of elements of $6 n+3$ type or $3 x(2 n+1)$ type, so all elements of S3 will be multiples of the number 3. This leads to conclude that series S3 consists of composites and no prime (number ' 3 ' being the only exception). A similar logic is that as all elements of S3 are having SADN as 3,6 or 9 leading to $\mathrm{S} 3$ elements beings multiples of 3 and hence being composites.

Hence prime numbers may belong to only S1 and S5. Additionally as mentioned above, series S1 has elements of type $6 n+1$ and series S5 has elements of type $6 n-1$. So this segregation of S1, S3 and S5 series also segregates the primes of types $6 n+1$ and $6 n-1$.

All $6 n+1$ type primes (and composites) will be only on series S1.

All 6n-1 type primes (and composites) will be only on series S5.

An additional explanation for classifying odd numbers into the above-mentioned three series is discussed below. 
Squares of all odd numbers bear a very specific relation with the number 24 . Specifically, when the square of the greatest common divisor (GCD) of odd numbers with SADN 1,2,4,5,7 and 8 is subtracted from the square of the odd number in question, the resultant number will be an integral multiple of the number ' 24 '. Since the GCD of odd numbers with SADN 1,2,4,5,7 and 8 is the number 1 , the 'square of the odd number' less 1 will be integral multiple of 24 . This may be expressed as follows:- If we denote odd numbers with SADN 1,2,4,5,7 and 8 as $\mathrm{d}$ and $\mathrm{k}$ is an integer, then

$$
d^{2}-1=24 k
$$

In case of odd numbers with SADN 3,6 and 9, since the GCD will be 3, the relation of such odd numbers with the number 24 would be expressed as follows-

If we denote odd numbers with SADN 3,6 and 9 as $\mathrm{m}$ and $\mathrm{k}$ as an integer then:-

$$
m^{2}-3^{2}=24 k \text { i.e. } m^{2}-9=24 k
$$

'Square of the first element' less 'one' of the S5 series is $5^{2}-1=24 \times 1$ i.e. $25-1=24$; while 'square of the first element' less 'one' of the S1 series is $7^{2}-1=24 \times 2$ i.e. $49-1=48=24 \times 2$ and in case of the $\mathrm{S} 3$ series, the square of $9^{2}-3^{2}=81-9=72=24 \times 3$

In order to obtain the quotient of the second term's square divided by 24 , a difference of 3 is to be added to the previous term. i.e. the quotient for the number 11 (second term of the S5 series, can be obtained as follows- $24 * 1+24 *(1+3)$. The quotient for the number 17 (third term of the S5 series can be derived as $24 * 5+24(4+7)$ and so on. Similarly in case of the S1 series, since the quotient for the first term 7 is 2 , the quotient for the second term 13 can be derived as $24 * 2+24(2+3)$. Quotient for the third term 19 can be derived as $24 * 7+24(5+3)$ and so on.

Applying the same rationale the quotient for the third term of the $\mathrm{S} 3$ series (15) can be derived as $24 * 3+24 *(3+3)$ and the quotient for the fourth term 21 can be derived as $24 * 9+24(6+3)$ and so on.

This shows that the starting points of the three series in terms of the quotient of the square of the first term less 'one' are different but the derivation of the quotient of subsequent numbers of the series is the same. Furthermore, if we horizontally sum up the quotients of the same element 
number of the three series, it leads us to the SADN identity i.e. 9. This is explained in the following table.

\begin{tabular}{|c|c|c|c|c|c|}
\hline $\begin{array}{l}\text { For } \mathrm{k}^{\text {th }} \\
\text { member of } \\
\text { S1,S2,S3 } \\
\text { where k= }\end{array}$ & $\begin{array}{l}\text { For } \\
\text { S1: } k^{2}- \\
(\mathrm{k}-1)^{2}\end{array}$ & $\begin{array}{l}\text { For S2: } \\
\mathrm{k}^{2}-(\mathrm{k}-1)^{2}\end{array}$ & $\begin{array}{l}\text { For S3: } \mathrm{k}^{2}- \\
(\mathrm{k}-1)^{2}\end{array}$ & $\begin{array}{l}24 \mathrm{xn}_{\mathrm{k}} \\
\text { where } \mathrm{n}_{\mathrm{k}} \\
\text { (represents } \\
\text { 'n' for a } \\
\text { given } \mathrm{k} \text { )= }\end{array}$ & $\begin{array}{l}\text { For a constant value } \\
\text { of } \\
\operatorname{SADN}\left(\sum \mathrm{n}_{\mathrm{k}}\right)=\text { identity } \\
\text { of } \mathrm{SADN}\end{array}$ \\
\hline \multirow[t]{3}{*}{1} & $\mathrm{Xxxx}$ & & & $\operatorname{xxxx}(-1)=8$ & \multirow[t]{3}{*}{$\operatorname{xxxx}(9)$} \\
\hline & & $\mathrm{Xxxx}$ & & $\operatorname{xxxx}(0)=9$ & \\
\hline & & & $\begin{array}{l}\operatorname{XXXX}(5)^{2}-(- \\
1)^{2}\end{array}$ & $\begin{array}{l}\operatorname{xxxx}(1) \\
\left(\mathrm{n}_{\mathrm{k}}=2 \mathrm{k}-1\right)\end{array}$ & \\
\hline \multirow[t]{3}{*}{2} & $7^{2}-1^{2}$ & & & 2 & \multirow[t]{3}{*}{9} \\
\hline & & $9^{2}-3^{2}$ & & 3 & \\
\hline & & & $11^{2}-5^{2}[2,5]$ & 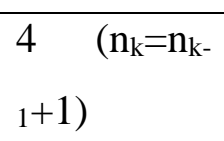 & \\
\hline \multirow[t]{3}{*}{3} & $\begin{array}{l}13^{2}-7^{2} \\
{[4,7]}\end{array}$ & & & 5 & \multirow[t]{3}{*}{9} \\
\hline & & $\begin{array}{l}15^{2}-9^{2} \\
{[6,9]}\end{array}$ & & 6 & \\
\hline & & & $17^{2}-11^{2}[8,2]$ & 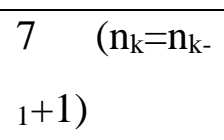 & \\
\hline \multirow[t]{3}{*}{4} & $\begin{array}{l}19^{2}- \\
13^{2} \\
{[1,4]}\end{array}$ & & & 8 & \multirow[t]{3}{*}{9} \\
\hline & & $\begin{array}{l}21^{2}-15^{2} \\
{[3,6]}\end{array}$ & & 9 & \\
\hline & & & $\begin{array}{l}23^{2}-17^{2} \\
{[5,8]}\end{array}$ & $\begin{array}{l}10\left(\mathrm{n}_{\mathrm{k}}=\mathrm{n}_{\mathrm{k}-}\right. \\
1+1)\end{array}$ & \\
\hline 5 & $\begin{array}{l}25^{2}- \\
19^{2}\end{array}$ & & & 11 & 9 \\
\hline
\end{tabular}




\begin{tabular}{|c|c|c|c|c|c|}
\hline & {$[7,1]$} & & & & \\
\hline & & $\begin{array}{l}27^{2}-21^{2} \\
{[9,3]}\end{array}$ & & 12 & \\
\hline & & & $\begin{array}{l}29^{2}-23^{2} \\
{[2,5]}\end{array}$ & $\begin{array}{l}13\left(\mathrm{n}_{\mathrm{k}}=\mathrm{n}_{\mathrm{k}-}\right. \\
1+1)\end{array}$ & \\
\hline 6 & $\begin{array}{l}31^{2}- \\
25^{2} \\
{[4,7]}\end{array}$ & & & 14 & 9 \\
\hline & & $\begin{array}{l}33^{2}-27^{2} \\
{[6,9]}\end{array}$ & & 15 & \\
\hline & & & $\begin{array}{l}35^{2}-29^{2} \\
{[8,2]}\end{array}$ & $\begin{array}{l}16\left(\mathrm{n}_{\mathrm{k}}=\mathrm{n}_{\mathrm{k}-}\right. \\
\left.{ }_{1}+1\right)\end{array}$ & \\
\hline 7 & $\begin{array}{l}37^{2}- \\
31^{2} \\
{[1,4]}\end{array}$ & & & 17 & 9 \\
\hline & & $\begin{array}{l}39^{2}-33^{2} \\
{[3,6]}\end{array}$ & & 18 & \\
\hline & & & $\begin{array}{l}41^{2}-35^{2} \\
{[5,8]}\end{array}$ & $\begin{array}{l}19\left(\mathrm{n}_{\mathrm{k}}=\mathrm{n}_{\mathrm{k}-}\right. \\
1+1)\end{array}$ & \\
\hline 8 & $\begin{array}{l}43^{2}- \\
37^{2} \\
{[7,1]}\end{array}$ & & & 20 & 9 \\
\hline & & $\begin{array}{l}45^{2}-39^{2} \\
{[9,3]}\end{array}$ & & 21 & \\
\hline & & & $\begin{array}{l}47^{2}-41^{2} \\
{[2,5]}\end{array}$ & $\begin{array}{l}22\left(\mathrm{n}_{\mathrm{k}}=\mathrm{n}_{\mathrm{k}-}\right. \\
1+1)\end{array}$ & \\
\hline 9 & $\begin{array}{l}49^{2}- \\
43^{2} \\
{[4,7]}\end{array}$ & & & 23 & 9 \\
\hline & & $51^{2}-45^{2}$ & & 24 & \\
\hline
\end{tabular}




\begin{tabular}{|c|c|c|c|c|c|}
\hline & & {$[6,9]$} & & & \\
\hline & & & $\begin{array}{l}53^{2}-47^{2} \\
{[8,2]}\end{array}$ & $\begin{array}{l}25\left(\mathrm{n}_{\mathrm{k}}=\mathrm{n}_{\mathrm{k}}\right. \\
\left.{ }_{1}+1\right)\end{array}$ & \\
\hline 10 & {$[1,4]$} & & & 26 & 9 \\
\hline & & {$[3,6]$} & & 27 & \\
\hline & & & {$[5,8]$} & $\begin{array}{l}28\left(\mathrm{n}_{\mathrm{k}}=\mathrm{n}_{\mathrm{k}-}\right. \\
1+1)\end{array}$ & \\
\hline
\end{tabular}

Table3: Of S1,S3 and S5: Periodic table in terms of SADN properties along rows and columns

Section-V: Deriving composite numbers of the $S 1$ and S5 series

- Multiplicative nature of SADN1,2,4,5,7,8

- Composite numbers of series S1 are intra-series products of elements of series S1 or intra-series products of elements of series S5

- Composite numbers of series S5 are inter-series products of elements of S1 and S5 An application of the property of SADN function being distributive over multiplication is the following table (Table 4) which leads to the condition for the elements of series S1 and S5.

\begin{tabular}{|c|c|c|c|c|c|}
\hline $\begin{array}{l}\text { Serie } \\
\mathrm{s}\end{array}$ & $\begin{array}{l}\text { SADN } \\
\text { of } \\
\text { element } \\
\text { s of } \\
\text { series }\end{array}$ & \multicolumn{4}{|c|}{$\begin{array}{l}\text { Possible combinations of SADN of divisors to yield the composite } \\
\text { number of particular SADN of series S1 or S5 }\end{array}$} \\
\hline $\mathrm{S} 1$ & SADN1 & $\begin{array}{l}\text { SADN1xSADN } \\
1\end{array}$ & $\begin{array}{l}\text { SADN2xSADN } \\
5\end{array}$ & $\begin{array}{l}\text { SADN4xSADN } \\
7\end{array}$ & $\begin{array}{l}\text { SADN8xSADN } \\
8\end{array}$ \\
\hline S1 & SADN7 & $\begin{array}{l}\text { SADN1xSADN } \\
7\end{array}$ & $\begin{array}{l}\text { SADN2xSADN } \\
8\end{array}$ & $\begin{array}{l}\text { SADN4xSADN } \\
4\end{array}$ & $\begin{array}{l}\text { SADN5xSADN } \\
5\end{array}$ \\
\hline S1 & SADN4 & $\begin{array}{l}\text { SADN1xSADN } \\
4\end{array}$ & $\begin{array}{l}\text { SADN2xSADN } \\
2\end{array}$ & $\begin{array}{l}\text { SADN5xSADN } \\
8\end{array}$ & $\begin{array}{l}\text { SADN7xSADN } \\
7\end{array}$ \\
\hline
\end{tabular}




\begin{tabular}{|l|l|l|l|l|l|}
\hline S5 & SADN5 & $\begin{array}{l}\text { SADN1xSADN } \\
5\end{array}$ & $\begin{array}{l}\text { SADN2xSADN } \\
7\end{array}$ & $\begin{array}{l}\text { SADN4xSADN } \\
8\end{array}$ & Xxxxxx \\
\hline S5 & SADN2 & $\begin{array}{l}\text { SADN1xSADN } \\
2\end{array}$ & $\begin{array}{l}\text { SADN4xSADN } \\
5\end{array}$ & $\begin{array}{l}\text { SADN7xSADN } \\
8\end{array}$ & Xxxxxx \\
& & SADN1xSADN & SADN2xSADN & SADN5xSADN & Xxxxxx \\
& & 4 & 7 & \\
\hline
\end{tabular}

Table4: Series and SADN of series-elements alongwith possible combinations of divisors

Comparison of Table2 and Table4 concludes that:-

(a) Composites of SADN1 can be obtained by product of $\operatorname{SADN}(1 \times 1)$ or $\operatorname{SADN}(2 \times 5)$ or $\operatorname{SADN}(4 \times 7)$ or $\operatorname{SADN}(8 \times 8)$. In this case the only three possible combinations of both the divisor elements (1 and 1 OR 2 and 5 OR 4 and 7 OR 8 and 8) are always elements of a single series (S1 or S5 or S1 or S5)

(b) Composites of SADN7 can be obtained by product of $\operatorname{SADN}(1 \times 7)$ or $\operatorname{SADN}(2 \times 8)$ or $\operatorname{SADN}(4 \times 4)$ or $\operatorname{SADN}(5 \times 5)$. In this case the only four possible combinations of both the divisor elements (1 and 7 OR 2 and 8 OR 4 and 4 OR 5 and 5) are always elements of a single series (S1 or S5 or S1 or S5)

(c) Composites of SADN4 can be obtained by product of $\operatorname{SADN}(1 \times 4)$ or $\operatorname{SADN}(2 \times 2)$ or $\operatorname{SADN}(5 \times 8)$ or $\operatorname{SADN}(7 \times 7)$. In this case the only four possible combinations of both the divisor elements ( 1 and 4 OR 2 and 2 OR 5 and 8 OR 7 and 7) are always elements of a single series ( $\mathrm{S} 1$ or $\mathrm{S} 5$ or $\mathrm{S} 5$ or $\mathrm{S} 1$ )

(d) Composites of SADN5 can be obtained by product of SADN(1x5) or SADN(2x7) or SADN $(4 \times 8)$. In this case the only three possible combinations of both the divisor elements (1 and 5 OR 2 and 7 OR 4 and 8) are always elements of two different series (S1 and S5)

(e) Composites of SADN2 can be obtained by product of SADN(1x2) or SADN(4x5) or SADN(7x8). In this case the only three possible combinations of both the divisor elements (1 and 2 OR 4 and 5 OR 7 and 8) are always elements of two different series (S1 and S5) 
(f) Composites of SADN8 can be obtained by product of $\operatorname{SADN}(1 \times 8)$ or $\operatorname{SADN}(2 \times 4)$ or $\operatorname{SADN}(5 \times 7)$. In this case the only three possible combinations of both the divisor elements (1 and 8 OR 2 and 4 OR 5 and 7) are always elements of two different series (S1 and S5)

Regarding seriesS1: The first three ( $a, b$ and $c$ )of above conclusions suggest that composite numbers of series $\mathrm{S} 1$ are either intra-series products of elements of series $\mathrm{S} 1$ or intra-series products of elements of series S5.

Regarding series S5: The next three (d, e and f) of above conclusions suggest that composite numbers of series S5 are always inter-series products of elements of S1 and S5.

\section{Section-VI Pattern of formation of composite numbers on the S1 and S5 series}

In context of Composites on S1 series:-

As composites contained in S1 are products of intra-series elements of S1 and S3, say products of intra-series elements of $\mathrm{S} 1$ are denoted by $\mathrm{C} 1$ and that of $\mathrm{S} 3$ are denoted by $\mathrm{C} 3$ :-

$C 1=[(6 n+1) \cdot(6 n+1)+(6 n+1) \cdot 6 n '] ;$ for each and every $n \in\{N\}$, there exists n' $\epsilon\{0, N\}$

Or $C 1=(6 n+1) \cdot[6(n+n ')+1]$; for each and every $n \in\{N\}$, there exists n' $\epsilon\{0, N\}$

Implies that $\mathrm{C} 1=\{49,91,133,175, \ldots\}+\{91,169,247,325,403, \ldots\}+\{133,247,361,475, \ldots\}$ $+\{175,325,475,625, \ldots\}+\ldots+\{\}+\ldots$ infinite sets of infinite terms

and $\quad C 3=\left[(6 n-1) \cdot(6 n-1)+(6 n-1) \cdot 6 n^{\prime}\right] ;$ for each and every $n \epsilon\{N\}$, there exists n' $\epsilon\{0, N\}$

Or C3 $=(6 n-1) \cdot\left[6\left(n+n^{\prime}\right)-1\right]$; for each and every $n \in\{N\}$, there exists $n ' \epsilon\{0, N\}$

Implies that $\mathrm{C} 3=\{25,55,85,115, \ldots\}+\{55,121,187, \ldots\}+\{85,187,289, \ldots\}+\{115,253,391, \ldots\}$ $+\ldots+\{\}+\ldots$ infinite sets of infinite terms

From above discussion: 


\section{Primes on series $\mathrm{S} 1=\mathrm{S} 1-$ composites of $\mathrm{S} 1=\mathrm{S1}-\mathrm{C} 1-\mathrm{C} 3$}

In context of Composites on S5 series:-

As composites contained in S5 are products of interseries elements of S1 and S5, say interseries products of S1 and S5 are denoted by $\mathrm{C} 15$,

$C 15^{\prime}=\left[5(6 n+1)+(6 n+1) .6 n^{\prime}\right] ;$ for each and every $n \in\{N\}$, there exists $n^{\prime} \epsilon\{0, N\}$

$C 15^{\prime}=(6 n+1) .\left(5+6 n^{\prime}\right)$; for each and every $n \in\{N\}$, there exists $n^{\prime} \epsilon\{0, N\}$

${\mathrm{C} 15^{\prime}}^{\prime}=\{35,77,119,161,203, \quad\}+\{65,143,221,299, \ldots\}+\ldots .+\{\}+\ldots\{\}+\ldots$

\section{Primes on series S5 = S5- composites of S5 = S5 - C15}

The pattern of formation of composite numbers on the S1 and S5 series as mentioned above has been confirmed both graphically (for limited values of integers) and also through a computer program (again, for limited values of integers).

\section{Section-VII}

\section{Conclusion}

The concept of SADN is very simple in its appearance but has very profound implications in understanding complex phenomenon like distribution of prime numbers.

The paper begins with the thought that the phenomenon of SADN has been occasionally identified but seldom employed to extensive mathematical applications. However, a detailed analysis of the properties of SADN in terms of fundamental mathematical functions like addition, subtraction and multiplication and application of the same in understanding the interrelationship between numbers reveals that there may be rather deep and profound implications in understanding 'numbers'. Based on the SADN of any odd number and the relation of the square of the number with the number '24' with specific reference to the division function, all odd numbers can be placed in three series -the S1 series comprising of odd numbers with SADN1, 4 and 7; the S3 series comprising of odd numbers with SADN 3, 6 and 9 and the S5 
series comprising of odd numbers with SADN 2,5 and 8. The paper brings out why the S3 series comprises exclusively of composite numbers while the S1 and S5 series consist of both prime and composite numbers. Further, the paper also brings out that composite numbers on the S1 and S5 series are products of the elements of the S1 and S5 series. More specifically, composite numbers on the S1 series can be derived as intra-series products of elements of the S1 and S5 series while composite numbers on the S5 series can be derived as inter-series products of the elements of the S1 and S5 series. Extending the analysis the paper also explains the pattern of formation of composite numbers on the S1 and S5 series. Based on the analysis the paper concludes that the concept of SADN is rather simple in its formulation but may have farreaching implications for number theory like decoding complex mathematical questions like understanding the distribution of prime numbers.

\section{Declaration of interests: None}

Funding: This research did not receive any specific grant from funding agencies in the public, commercial, or not-for-profit sectors.

\section{Acknowledgements:}

Authors acknowledge the help provided by Mr. Abhishek Dubey, in confirming the results of the study through computer program. Thanks are also due to (unsuccessful) efforts made by Ms. Ojal Bhatnagar and Mr. Atharv Yeole in helping authors with the computer program to verify the results of this study. Special thanks to Mr. Azhar Shaikh for bringing to notice of author (MK) that the proofs of properties of SADN were missing in the earlier version of present paper.

\section{Bibliography:}

[1] Courant, R. and Robbins, H., What is Mathematics, Oxford University Press(2016), ISBN10: 0-19-568710-8

[2] Sen S., 103 Mathematical Theorems, Axioms, Paradoxes and Stuff, Scholastic(2012), ISBN13:978-81-7655-866-2

[3] Hogben, L., Mathematics for the Million, W W Nortan \& Company(2018), ISBN:978-0-393-31071-9 